\title{
ESTIMAÇÃo dE PARÂMETROS PCOs E PCVs POR INTEGRAÇÃo NUMÉRICA
}

\section{Estimation of PCO and PCV Parameters by Numerical Integration}

\author{
Luiz Fernando Macedo Morescki Junior ${ }^{1,2}$ \\ Luiz Danilo Damasceno Ferreira ${ }^{2}$
}

Instituto Federal de Educação, Ciência e Tecnologia de Santa Catarina - Campus Jaraguá do Sul. Rua Getúlio Vargas, 830, Centro. Jaraguá do Sul, Santa Catarina, Brasil ${ }^{1}$. E-mail:luizm@ifsc.edu.br

Universidade Federal do Paraná, Programa de Pós-Graduação em Ciências Geodésicas. Avenida Coronel Francisco Heráclito dos Santos, 210, Jardim das Américas. Curitiba, Brasil². E-mail:luizdaniloferreira@gmail.com

\section{Resumo:}

Para melhorar a qualidade da estimativa das coordenadas tridimensionais de um ponto na superfície da Terra, é necessário que os erros decorrentes da variação do centro de fase da antena do receptor GNSS sejam conhecidos e modelados. A modelagem depende do conhecimento dos parâmetros associados à localização do centro de fase, $\mathrm{PCO}$ e PCV, os quais são determinados mediante procedimentos de calibração em caráter absoluto ou relativo. Neste artigo são utilizados dados de calibrações relativas da antena Leica AX 1202GG conduzidas pela BCAL/UFPR utilizando o programa de calibração WaSoft/Kalib. Parâmetros PCOs, estabelecidos pelo programa, são considerados como condições iniciais para a propagação dos parâmetros por meio de integração numérica, desde uma data inicial até uma data final. Com base nos parâmetros PCOs propagados, novos parâmetros PCVs também são estimados. Os parâmetros preditos são comparados com os parâmetros calibrados para a mesma época. Os resultados mostram discrepâncias máximas de pouco mais de $4 \mathrm{~mm}$ para a componente vertical do vetor PCO e de 5,5 mm para as PCVs quando são consideradas campanhas afetadas por precipitação. Utilizando campanhas sem ocorrência de precipitação, os resultados máximos para as discrepâncias dos parâmetros PCO e PCV não ultrapassam 1,5 e 2,2 mm, respectivamente.

Palavras-chave: Parâmetros PCOs e PCVs, Integração Numérica, WaSoft/Kalib.

\section{Abstract:}

For improving the quality of the estimation of three-dimensional coordinates of a point on the surface of the Earth, it is necessary that the errors derived from the variation of the GNSS receiver antenna's phase center be known and modeled. The modeling depends on the knowledge of parameters associated to the location of the phase center, PCO and PCV, which are determined through calibration proceedings in absolute or relative character. In this work, 
relative calibrations data, conducted by BCAL/UFPR, of the Leica AX 1202GG antenna, that use the WaSoft/Kalib calibration program, were utilized. PCO parameters, established by the program, are considered as initial conditions and propagated, by numeric integration, from an initial date until a final date. Based on propagated PCO parameters, new PCV parameters are also determined for the antenna. The predicted parameters are compared to the calibrated parameters for the same epoch. The results show maximum discrepancies just over $4 \mathrm{~mm}$ for the vertical component PCO vector and $5.5 \mathrm{~mm}$ for the PCV when calibration campaigns affected by precipitation are considered. Utilizing campaigns with no precipitation occurrence, the maximum results to the PCO and PCV discrepancies do not exceed 1.5 and $2.2 \mathrm{~mm}$, respectively.

Keywords: PCO and PCV Parameters, Numerical Integration, WaSoft/Kalib.

\section{Introdução}

Uma das aplicações dos sistemas de posicionamento por satélites é a localização de pontos sobre a superfície da Terra com a determinação de coordenadas tridimensionais ao nível do milímetro. Para que tal qualidade no posicionamento possa ser atingida, é necessário o conhecimento e modelagem dos erros sistemáticos que afetam as medidas.

A acurácia nas observações é degradada por algumas fontes de erros cujos efeitos necessitam ser tratados de maneira adequada para que o sistema desempenhe com máxima eficácia as suas funções.

As principais fontes de erros podem ser reunidas em três grupos:

- erros provenientes das posições dos satélites;

- erros associados à propagação do sinal;

- erros relacionados ao receptor.

Destacando o último grupo, os erros que dizem respeito à localização do centro de fase da antena têm caráter sistemático e torna-se imprescindível o conhecimento e modelagem das incertezas relacionadas às suas variações quando a acurácia do posicionamento necessita alcançar patamares milimétricos (Fang et al., 1998; Monico, 2008; Huinca, 2009; Krueger et al., 2012).

Nas aplicações geodésicas que envolvem alta acurácia, como os trabalhos em geodinâmica, é importante conhecer com a máxima exatidão possível a posição do centro de fase da antena do receptor GNSS (Global Navigation Satellite System), uma vez que a posição é estimada, justamente, onde se encontra a antena. $\mathrm{O}$ estudo das variações do centro de fase de uma antena GNSS é de fundamental relevância e vem sendo objeto de intensa pesquisa (Görres et al., 2006; El-Hattab, 2013).

$\mathrm{O}$ conhecimento completo do centro de fase da antena está relacionado à determinação do vetor PCO (Phase Center Offset) e das PCVs (Phaser Center Variations), parâmetros que são estabelecidos mediante o processo de calibração de antenas. Os valores determinados fazem com que os erros ocasionados pelas variações do centro de fase sejam minimizados ou mesmo eliminados por completo quando utilizados no processamento de dados (Rothacher, 1995; Menge, 2003; Zeimetz e Kuhlmann, 2008).

Os parâmetros PCOs e PCVs participam de forma decisiva na determinação da componente vertical do posicionamento, causando variações que podem chegar à ordem centimétrica quando 
são comparadas coordenadas obtidas empregando-se os parâmetros com as coordenadas estabelecidas sem a aplicação dos mesmos. Em Mader (1999), comenta-se que a magnitude das variações pode atingir valores de até $10 \mathrm{~cm}$.

Trabalhos como Menge et al. (1998), Krueger et al. (2007) e Huinca et al. (2012) mostram a importância da execução dos procedimentos de calibração das antenas com o objetivo de obtenção dos parâmetros próprios precedendo à realização de tarefas que exijam posicionamento acurado.

A aplicação dos métodos de calibração de antenas exige o emprego de equipamentos específicos e locais adequados durante o intervalo de tempo em que os procedimentos são realizados. Assim, o usuário que necessita levar em consideração os parâmetros próprios do centro de fase da antena, deve executar o processo de calibração ou enviar a antena para ser calibrada em instituições que apresentem os recursos necessários para os procedimentos. Mesmo que já possua os parâmetros, sugere-se que o usuário os atualize periodicamente. Em Huinca (2014), recomenda-se que o usuário realize um acompanhamento anual dos parâmetros.

Nesta pesquisa, propõe-se que os parâmetros do centro de fase de uma antena GNSS sejam estimados por meio de integração numérica envolvendo as componentes do vetor PCO, consideradas como condições iniciais, bem como as respectivas taxas de variação temporal. Para isso, utilizam-se, os parâmetros calibrados do centro de fase da antena fornecidos por campanhas de calibração em que o programa WaSoft/Kalib é empregado.

\section{Centro de fase da antena}

Se a antena for considerada como uma fonte pontual emissora de sinais, as frentes de onda que dela emanam teriam, na região de campo distante, a forma de esferas concêntricas ao ponto tomado como fonte, tal ponto é chamado de centro de fase. Cada esfera consiste em elementos de campo possuidores de mesma fase, portanto, são consideradas superfícies equifases (Born e Wolf, 1980; Hecht, 1990).

O IEEE (Institute of Electrical and Electronics Engineers) estabelece em seu documento, Standard Definitions of Terms for Antennas (IEEE Std. 145-1983), que o centro de fase de uma antena é a localização de um ponto associado a uma antena de tal forma que, se for tomado como centro de uma esfera da qual o raio se estende ao campo distante, a fase de um componente do campo sobre a superfície da esfera de radiação é essencialmente constante, pelo menos sobre a porção da superfície onde a radiação é significante. No entanto, para uma antena real, as dimensões não são zero e, desta forma, as superfícies equifases deixariam de ser esféricas (de Jong, 1982).

Pelo teorema da reciprocidade, numa situação de recepção a antena apresenta características idênticas às apresentadas por ela numa situação de emissão (Markov, 1965; Kraus, 1983; Balanis, 2005). Desta forma, o centro de fase de uma antena, empregada na recepção de energia pode ser entendido como o ponto da antena em que o sinal é coletado.

Na prática, nenhuma antena é uma fonte pontual capaz de emitir frentes de ondas esféricas ideais. A antena é uma fonte de radiação de tamanho finito o que produz frentes de onda que não serão totalmente esféricas, mas sim distorcidas. Neste caso, cabe analisar a frente de onda como 
uma série de pequenas porções esféricas com um correspondente centro para cada uma delas. Um único centro de fase válido para todas as porções esféricas não existe, pois é dependente da direção em que se encontra a porção esférica. O centro de fase, então, não é constante, mas dependente do ângulo de observação, Figura 1. Portanto, a definição teórica de centro de fase deve ser transformada em uma definição de caráter prático (Bartels, 1997).

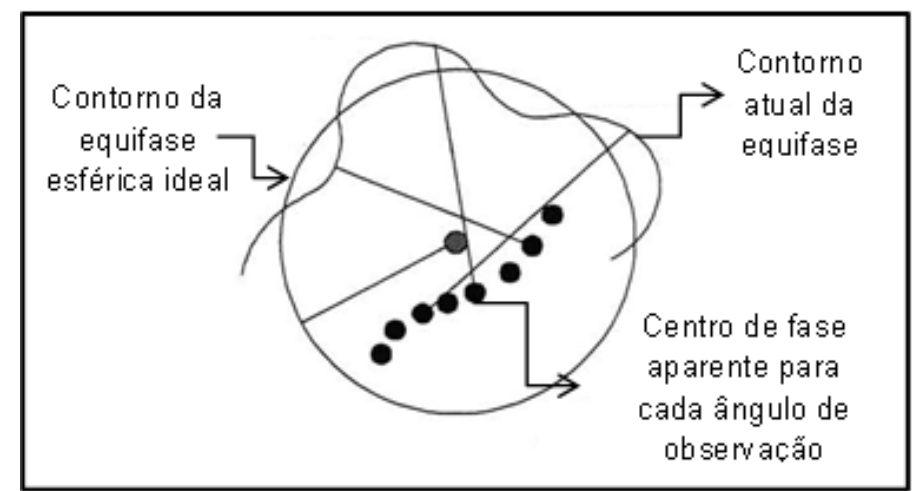

Figura 1: Centro de fase da antena dependente do ângulo de observação.

Fonte: Adaptado Bartels (1997).

\subsection{Variações do Centro de Fase da Antena}

Da mesma forma em que não existe um único centro de fase válido para todos os ângulos de elevação e de azimute a partir de uma antena emissora, pelo teorema da reciprocidade, não há também um único centro de fase válido para todas as direções, no caso de uma antena que atua na recepção de sinais. Desta forma, há uma mudança de posição do ponto em que o sinal eletromagnético é coletado pela antena.

O posicionamento por GNSS refere-se ao centro de fase eletrônico da antena, o qual varia com a intensidade e direção dos sinais incidentes (Seeber, 1993).

Para a descrição completa do centro de fase da antena deve-se, portanto, considerar que a incidência de sinais provenientes de diversas direções definem um centro de fase elétrico médio da antena, ou, simplesmente, centro de fase médio (CFM). De maneira equivalente, define-se um centro de fase elétrico, ou, apenas, centro de fase $(\mathrm{CF})$ que dependerá da direção de incidência de cada sinal.

Tanto o CFM quanto o CF são referenciados ao chamado ponto de referência da antena ou ARP (Antenna Reference Point). O IGS (International GNSS Service) define o ARP como sendo o ponto físico formado pela intersecção do centro da rosca de centragem forçada, que se localiza na superfície mais inferior da antena, com o eixo de simetria do equipamento. O ARP é a origem do sistema de referência da antena e relaciona-se com os parâmetros que estabelecem o centro de fase.

A caracterização da posição do CFM é definida pelo vetor PCO (Phase Center Offset), a partir do ARP. Além disso, para cada direção, existirá um centro de fase instantâneo CF. A distância entre o CFM e o CF, em uma determinada direção, caracteriza a variação do centro de fase, PCV 
(Phase Center Variation), com dependência do azimute $(\alpha)$ e do ângulo zenital $(\theta)$ do sinal incidente, $\operatorname{PCV}(\alpha, \theta)$, conforme a Figura 2.

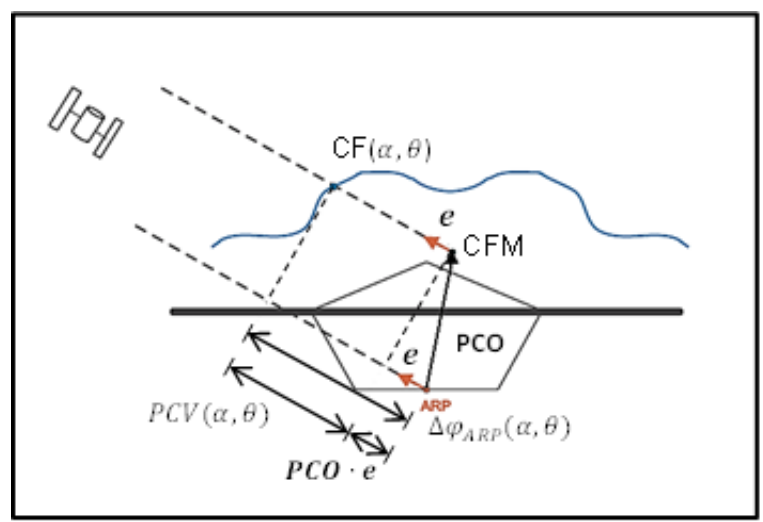

Figura 2: Relação entre o vetor PCO e as PCVs.

Fonte: Adaptado Menge (2003).

Em princípio, o CFM é uma aproximação do comportamento real do centro de fase da antena, ao mesmo tempo em que é o centro de uma superfície esférica que corresponde ao diagrama de fase médio ou ideal (isotrópico) que mais se aproxima do diagrama de fase efetivo (Menge, 2003). Assim, as PCVs podem ser interpretadas como um adiantamento ou um atraso que ocorre no diagrama de fase atual ou real em relação ao diagrama de fase ideal, o que resulta em distâncias remanescentes entre os diagramas representantes dos padrões.

As considerações feitas ao vetor $\mathrm{PCO}$ e às $\mathrm{PCV}$ s produzem correções que devem ser vinculadas ao ARP de modo que para cada vetor PCO um único conjunto de parâmetros PCVs estão associados a ele, ou seja, dois conjuntos distintos de parâmetros PCVs não podem estar associados ao mesmo vetor PCO. Menge (2003) apresenta a fórmula para o cálculo das correções, através do produto interno entre o vetor PCO e o vetor unitário na direção de recepção do sinal somada à respectiva PCV da referente direção, de modo que:

$$
\Delta \varphi_{A R P}(\alpha, \theta)=\boldsymbol{P C} \boldsymbol{O} \cdot \boldsymbol{e}+\operatorname{PCV}(\alpha, \theta),
$$

onde $\boldsymbol{e}$ diz respeito ao vetor unitário na direção do sinal incidente.

A Equação 1 representa um conjunto original de correções, $\Delta \varphi_{A R P}(\alpha, \theta)$, que se caracteriza por um vetor PCO e uma função que leva em consideração as $\operatorname{PCVs~direcionais,~} \operatorname{PCV}(\alpha, \theta)$. Cada conjunto pode ser transformado em um novo conjunto de correções, com um novo vetor PCO e novas PCVs que fornecerão os mesmos resultados do conjunto original quando aplicados no processamento de dados GPS (Rothacher et al., 1995).

Portanto, para uma mesma antena, infinitos conjuntos de parâmetros PCOs e PCVs produzem as mesmas correções para uma mesma onda portadora (Menge, 2003):

$$
\begin{aligned}
\Delta \varphi_{A R P}^{f, n}(\alpha, \theta) & =\boldsymbol{P C O}^{f, 1} \cdot \boldsymbol{e}+P C V^{f, 1}(\alpha, \theta) \\
& =\boldsymbol{P C} \boldsymbol{O}^{f, 2} \cdot \boldsymbol{e}+P C V^{f, 2}(\alpha, \theta) \\
& =\boldsymbol{P C} \boldsymbol{O}^{f, 3} \cdot \boldsymbol{e}+P C V^{f, 3}(\alpha, \theta)
\end{aligned}
$$


onde $f$ diz respeito à frequência da onda portadora e $n$ refere-se ao número do conjunto parâmetros.

Assim, considerando dois conjuntos de parâmetros para uma mesma onda portadora, cada um composto pelo vetor PCO e as PCVs a ele associadas, as Equações 2 podem ser escritas como (Menge, 2003):

$$
\boldsymbol{P C} \boldsymbol{O}^{f, 1} \cdot \boldsymbol{e}+P C V^{f, 1}(\alpha, \theta)=\boldsymbol{P C} \boldsymbol{O}^{f, 2} \cdot \boldsymbol{e}+P C V^{f, 2}(\alpha, \theta)
$$

\section{Materiais e método}

Os dados utilizados para a referente proposta metodológica são os parâmetros do centro de fase da antena Leica AX 1202GG que foram estabelecidos por campanhas de calibração conduzidas pela BCAL/UFPR (Base de Calibração da Universidade Federal do Paraná), primeira base de calibração de antenas GNSS do Brasil (Freiberger Junior, 2007; Krueger et al., 2007; Huinca, 2009; Huinca et al., 2012). O processamento dos dados para a determinação dos parâmetros de calibração foi conduzido no programa WaSoft/Kalib, em que se empregou a antena Leica AR25 como antena de referência. Ambas as antenas funcionaram conectadas ao receptor Leica GPS 1200 .

A partir de duas campanhas de calibração, definidas como campanha primária e campanha secundária, tomadas em ordem cronológica crescente, determina-se a taxa de variação temporal de cada componente do vetor PCO. Em seguida, as componentes são propagadas por integração numérica pelo método Runge-Kutta de ordem 7(8) com coeficientes de Fehlberg (Fehlberg, 1968). O passo de integração é fixo e a propagação é realizada até uma data específica, caracterizando assim, o vetor PCO propagado. Após a determinação do vetor PCO propagado, determinam-se os parâmetros PCVs a ele associados.

Como objetivo de validação do método, os parâmetros propagados (vetor PCO e os respectivos parâmetros PCV) serão comparados àqueles estabelecidos pelo programa WaSoft/Kalib para a mesma época.

\subsection{Propagação das Componentes do Vetor PCO}

O vetor PCO tem origem no ARP e é conhecido por suas componentes Este, Norte e Vertical (E, $\mathrm{N}, \mathrm{V})$.

Analisando-se o comportamento das componentes do vetor PCO através de campanhas de calibração diferentes, verifica-se que existe uma variação temporal aproximadamente linear envolvendo duas campanhas distintas, Assim, define-se uma taxa de variação temporal para o vetor PCO entre duas campanhas de calibração quaisquer como: 


$$
\frac{d \boldsymbol{P}}{d t}=\frac{\Delta \boldsymbol{P}}{\Delta t}=\frac{\boldsymbol{P}_{2}-\boldsymbol{P}_{1}}{t_{2}-t_{1}},
$$

onde: $\boldsymbol{P}_{1}=$ vetor PCO relativo à campanha primária;

$\boldsymbol{P}_{2}=$ vetor $\mathrm{PCO}$ relativo à campanha secundária;

$t_{1}=$ tempo de início da primeira campanha calculada em Dias Julianos Modificados (DJM);

$t_{2}=$ tempo de encerramento da segunda campanha em (DJM).

Representando-se o sistema de referência da antena conforme Figura 3,

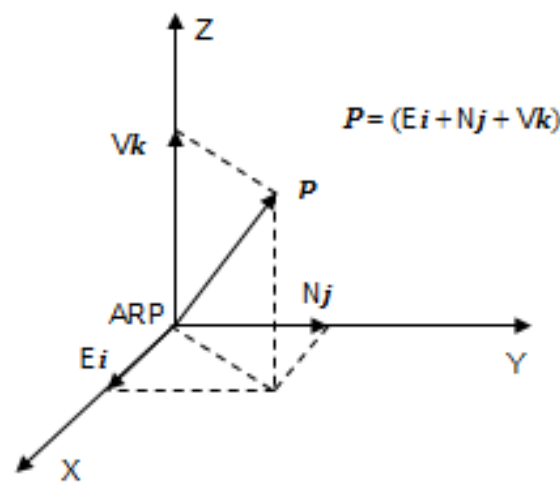

Figura 3: Vetor PCO representado no referencial da antena.

escrevem-se $\boldsymbol{P}_{1}$ e $\boldsymbol{P}_{2}$ como:

$$
\begin{aligned}
& \boldsymbol{P}_{1}=\left(E_{1} \boldsymbol{i}+N_{1} \boldsymbol{j}+V_{1} \boldsymbol{k}\right), \\
& \boldsymbol{P}_{2}=\left(E_{2} \boldsymbol{i}+N_{2} \boldsymbol{j}+V_{2} \boldsymbol{k}\right) .
\end{aligned}
$$

Uma vez considerado o comportamento linear do vetor PCO e de suas componentes em relação ao tempo, escreve-se:

$$
\boldsymbol{P}_{i}^{\prime}=\boldsymbol{P}_{i-1}^{\prime}+\frac{d \boldsymbol{P}}{d t} \Delta t
$$

onde: o subíndice $(i)$ indica o número de iterações. Portanto, para $i=1$ tem-se:

$\boldsymbol{P}^{\prime}{ }_{0}=$ PCO inicial, o qual coincide com o vetor PCO da campanha primária.

Os demais elementos são identificados como:

$$
\begin{aligned}
& \boldsymbol{P}^{\prime}{ }_{1}=\text { vetor PCO propagado; } \\
& \frac{d \boldsymbol{P}}{d t}=\text { vetor taxa de variação } \mathrm{PCO} \text { e } \\
& \Delta t=\text { tempo de propagação, em (DJM). }
\end{aligned}
$$

Exemplificando-se o comportamento linear das componentes do vetor PCO, integram-se numericamente as Equações 7, e apresentam-se no gráfico da Figura 4 os valores das três componentes, para a onda portadora L1, ao longo do período de integração, iniciado às $19 \mathrm{~h}$ 
04min 30s (UTC) (Universal Time Coordinated) do dia 12 de maio de 2011 até às 14h 34min. 00s (UTC) do dia 01 de setembro de 2011.

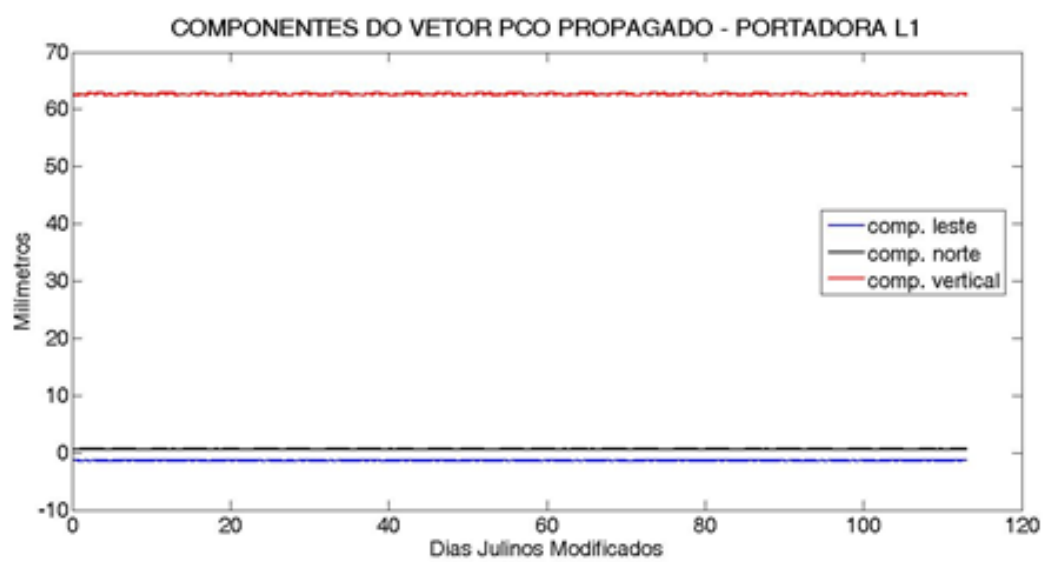

Figura 4: Comportamento das componentes do vetor PCO propagado - onda portadora L1.

O comportamento das componentes do vetor PCO propagado relativo à onda portadora L2 se assemelha ao apresentado pelas componentes do vetor PCO referente à onda portadora L1.

As oscilações apresentadas pelas componentes representam o comportamento dos valores no decorrer do processo de integração numérica em virtude do passo de integração adotado.

Mediante o valor propagado do vetor PCO, determinam-se os parâmetros PCVs associados utilizando-se a Equação 3. O procedimento pode ser encontrado em Morescki Junior e Ferreira (2014).

Finalmente, comparam-se os parâmetros PCOs e PCVs determinados pelo método apresentado com os parâmetros calibrados da antena. Na comparação entre os parâmetros PCVs propagados com os parâmetros PCVs calibrados considera-se que os respectivos PCOs são compatibilizados, uma vez que que as correções são iguais entre os conjuntos de parâmetros.

\subsection{Parâmetros de Calibração Utilizados nos Experimentos}

A metodologia proposta utilizou como dados resultados de campanhas de calibração da antena Leica AX 1202GG realizadas nos meses de maio, agosto, setembro e outubro de 2011 e abril de 2012 com o emprego do programa WaSoft/Kalib que fornece, além dos parâmetros de calibração da antena, também informações sobre a data, o horário de início UTC e o tempo de duração. As informações sobre a data, o início e a duração das campanhas fornecedoras dos parâmetros utilizados nos experimentos estão apresentadas no Quadro 1. 
Quadro 1: Data, início e duração das campanhas fornecedoras dos parâmetros de calibração.

\begin{tabular}{|c|c|c|c|}
\hline Campanha & Data (dd/mm/aa) & Início (hh:mm:ss - UTC) & Duração (h) \\
\hline 1 & $12 / 05 / 2011$ & $19: 04: 30$ & 24,1 \\
\hline 2 & $13 / 05 / 2011$ & $19: 33: 45$ & 24,7 \\
\hline 3 & $14 / 05 / 2011$ & $20: 32: 45$ & 40,3 \\
\hline 4 & $30 / 08 / 2011$ & $19: 19: 45$ & 24,2 \\
\hline 5 & $01 / 09 / 2011$ & $13: 05: 15$ & 24,4 \\
\hline 6 & $02 / 09 / 2011$ & $14: 34: 00$ & 29,8 \\
\hline 7 & $03 / 09 / 2011$ & $20: 54: 15$ & 39,5 \\
\hline 8 & $05 / 09 / 2011$ & $13: 03: 30$ & 24,2 \\
\hline 9 & $26 / 09 / 2011$ & $15: 07: 30$ & 24,4 \\
\hline 10 & $27 / 09 / 2011$ & $15: 39: 30$ & 24,8 \\
\hline 11 & $28 / 09 / 2011$ & $17: 57: 00$ & 24,7 \\
\hline 12 & $01 / 10 / 2011$ & $17: 17: 30$ & 42,6 \\
\hline 13 & $03 / 04 / 2012$ & $12: 35: 30$ & 24,0 \\
\hline
\end{tabular}

Os parâmetros do centro de fase determinados pelas campanhas de calibração são apresentados no Quadro 2.

Quadro 2: Parâmetros do centro de fase determinados pelas campanhas de calibração.

\begin{tabular}{|c|c|c|c|c|c|c|}
\hline \multirow{2}{*}{ Campanha } & \multicolumn{4}{|c|}{ Portadora Ll (mm) } & \multicolumn{3}{|c|}{ Portadora L2 (mm) } \\
\cline { 2 - 7 } & $\mathbf{E}$ & $\mathbf{N}$ & $\mathbf{V}$ & $\mathbf{E}$ & $\mathbf{N}$ & $\mathbf{V}$ \\
\hline 1 & $-1,25$ & 0,69 & 62,70 & 0,68 & 0,07 & 63,12 \\
\hline 2 & $-1,32$ & 0,66 & 62,69 & 0,68 & $-0,10$ & 62,99 \\
\hline 3 & $-1,60$ & 1,10 & 65,54 & 0,68 & $-0,37$ & 63,78 \\
\hline 4 & $-0,30$ & 1,89 & 65,52 & 0,46 & $-0,77$ & 64,36 \\
\hline 5 & $-0,29$ & 1,45 & 63,85 & 0,31 & $-0,55$ & 63,95 \\
\hline 6 & $-0,24$ & 1,56 & 63,22 & 0,30 & $-0,75$ & 63,56 \\
\hline 7 & $-0,19$ & 1,39 & 63,59 & 0,30 & $-0,73$ & 63,65 \\
\hline 8 & $-0,16$ & 1,51 & 63,36 & 0,23 & $-0,66$ & 63,86 \\
\hline 9 & $-0,08$ & 1,52 & 62,94 & 0,17 & $-0,63$ & 63,26 \\
\hline 10 & $-0,89$ & 1,29 & 62,59 & 0,50 & $-0,41$ & 62,70 \\
\hline 11 & $-0,80$ & 1,20 & 62,98 & 0,48 & $-0,41$ & 63,19 \\
\hline 12 & $-0,97$ & 1,21 & 64,38 & 0,45 & $-0,41$ & 63,97 \\
\hline 13 & $-0,54$ & 1,42 & 63,55 & 0,34 & $-0,52$ & 64,50 \\
\hline
\end{tabular}

Foram realizados 22 testes considerando, para cada um deles, dados de duas campanhas de calibração dentre as 13 disponíveis. Após a escolha de duas campanhas, a campanha com a data mais antiga entre elas foi denominada de Campanha Primária e os respectivos parâmetros PCOs foram considerados como condições iniciais para a propagação. A campanha com a data mais recente, entre as duas, foi denominada de Campanha Secundária cujos parâmetros PCO foram utilizados para o cálculo da respectiva taxa de variação temporal. Em seguida, os parâmetros PCOs são propagados até a data final de propagação estabelecida em cada teste e, então, são determinados os parâmetros PCVs associados ao PCO propagado. A escolha das duas 
campanhas utilizadas em cada teste foi procedida de forma aleatória. Um esquema do procedimento descrito é mostrado na Figura 5.

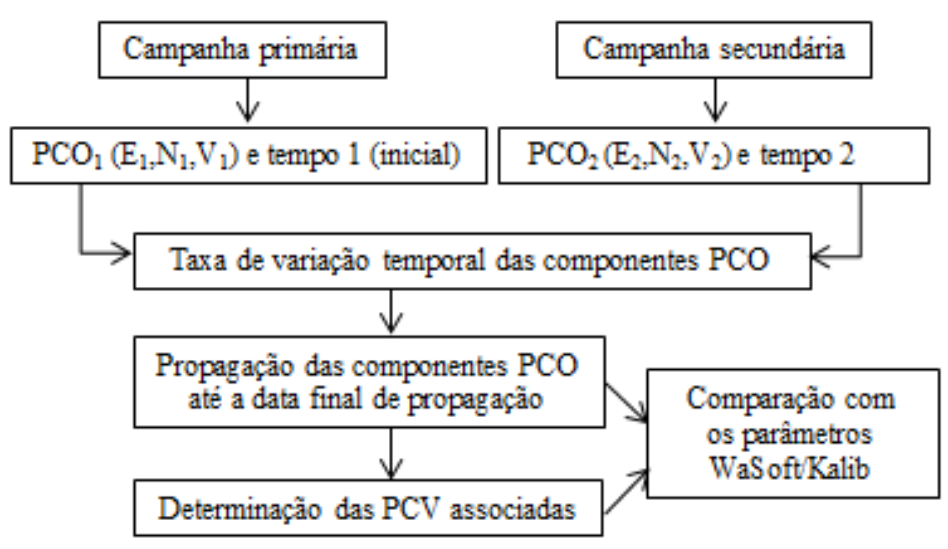

Figura 5: Esquema que demonstra os procedimentos realizados nos testes.

Evidentemente, como objetiva-se a comparação entre parâmetros propagados e calibrados, a data final de propagação empregada em cada teste deve coincidir com a data de uma campanha de calibração com data mais recente àquela estabelecida pela Campanha Secundária.

Nos testes 21 e 22, define-se um dia entre a data inicial e data final de uma propagação com a denominação de Data Intermediária, para a qual os parâmetros atribuídos pela propagação numérica até então possam ser comparados aos parâmetros estabelecidos pela campanha de calibração para o mesmo dia. A Figura 6 mostra um esquema deste procedimento.

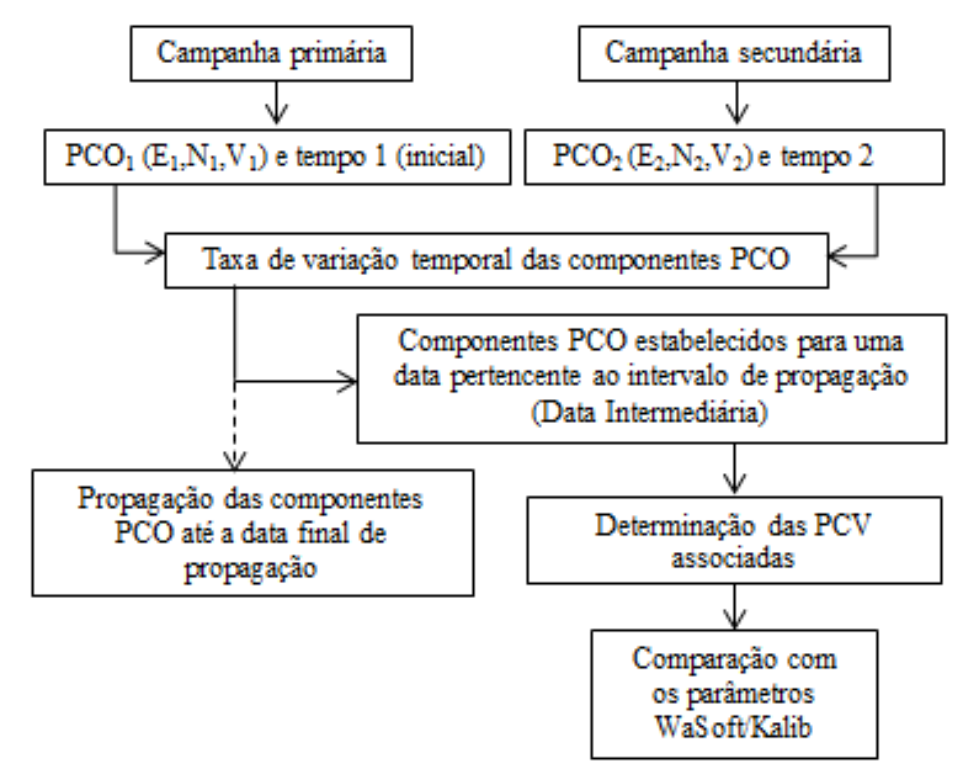

Figura 6: Esquema que demonstra os procedimentos realizados nos testes 21 e 22. 
As campanhas envolvidas até o teste 20, bem como o dia para o qual os parâmetros foram propagados (data final de propagação) são apresentados no Quadro 3.

Quadro 3: Campanhas consideradas até o teste 20.

\begin{tabular}{|c|c|c|c|}
\hline Teste & $\begin{array}{c}\text { Campanha } \\
\text { Primária }\end{array}$ & $\begin{array}{c}\text { Campanha } \\
\text { Secundária }\end{array}$ & $\begin{array}{c}\text { Data } \\
\text { final de } \\
\text { propagação }\end{array}$ \\
\hline 1 & 1 & 2 & $01-09-2011$ \\
\hline 2 & 1 & 2 & $02-09-2011$ \\
\hline 3 & 4 & 5 & $26-09-2011$ \\
\hline 4 & 4 & 5 & $27-09-2011$ \\
\hline 5 & 1 & 2 & $28-09-2011$ \\
\hline 6 & 4 & 5 & $28-09-2011$ \\
\hline 7 & 5 & 6 & $28-09-2011$ \\
\hline 8 & 5 & 8 & $28-09-2011$ \\
\hline 9 & 5 & 6 & $01-10-2011$ \\
\hline 10 & 5 & 7 & $17-02-2012$ \\
\hline 11 & 5 & 9 & $17-02-2012$ \\
\hline 12 & 9 & 11 & $17-02-2012$ \\
\hline 13 & 1 & 2 & $13-02-2012$ \\
\hline 14 & 1 & 3 & $13-02-2012$ \\
\hline 15 & 5 & 7 & $13-02-2012$ \\
\hline 16 & 5 & 9 & $13-02-2012$ \\
\hline 17 & 9 & 11 & $13-02-2012$ \\
\hline 18 & 9 & 11 & $09-03-2012$ \\
\hline 19 & 1 & 2 & $03-04-2012$ \\
\hline 20 & 9 & 11 & $03-04-2012$ \\
\hline
\end{tabular}

As campanhas envolvidas nos testes 21 e 22, a data final de propagação e a data intermediária utilizada são apresentadas no Quadro 4.

Quadro 4 : Campanhas consideradas pelos testes 21 e 22.

\begin{tabular}{|c|c|c|c|c|}
\hline Teste & $\begin{array}{c}\text { Campanha } \\
\text { Primária }\end{array}$ & $\begin{array}{c}\text { Campanha } \\
\text { Secundária }\end{array}$ & $\begin{array}{c}\text { Data } \\
\text { final de } \\
\text { propagação }\end{array}$ & Data intermediária \\
\hline 21 & 1 & 2 & $28-09-2011$ & $05-09-2011$ \\
\hline 22 & 5 & 6 & $01-10-2011$ & $28-09-2011$ \\
\hline
\end{tabular}




\section{Resultados}

Os resultados alcançados nos testes realizados têm como base as componentes do vetor PCO estimadas por propagação numérica. Desta forma, foram estimados 22 pares de vetores PCOs para a antena avaliada. Os valores obtidos em cada teste são apresentados na Tabela 1.

Tabela 1: Resultados das componentes propagadas.

\begin{tabular}{c|c|c|c|c|c|c}
\cline { 2 - 7 } \multicolumn{2}{c}{ Vetor PCO - Ll (mm) } & \multicolumn{3}{|c}{ Vetor PCO - L2 (mm) } \\
\hline Teste & E & N & V & E & N & V \\
\hline 1 & $-1,38$ & 0,63 & 62,56 & 0,68 & $-0,25$ & 62,76 \\
\hline 2 & $-1,31$ & 0,66 & 62,48 & 0,68 & $-0,08$ & 62,80 \\
\hline 3 & $-0,30$ & 1,74 & 65,09 & 0,41 & $-0,70$ & 64,35 \\
\hline 4 & $-0,28$ & 1,01 & 62,15 & 0,16 & $-0,33$ & 63,51 \\
\hline 5 & $-1,38$ & 0,63 & 62,57 & 0,68 & $-0,25$ & 62,76 \\
\hline 6 & $-0,30$ & 1,76 & 64,95 & 0,42 & $-0,71$ & 64,14 \\
\hline 7 & $-0,20$ & 1,65 & 62,84 & 0,29 & $-0,91$ & 63,37 \\
\hline 8 & $-0,06$ & 1,56 & 63,03 & 0,17 & $-0,75$ & 63,85 \\
\hline 9 & $-0,28$ & 1,48 & 63,84 & 0,31 & $-0,60$ & 64,00 \\
\hline 10 & $-0,10$ & 1,34 & 63,28 & 0,29 & $-0,88$ & 63,31 \\
\hline 11 & $-0,02$ & 1,54 & 62,65 & 0,13 & $-0,65$ & 63,03 \\
\hline 12 & $-0,26$ & 1,44 & 62,86 & 0,25 & $-0,57$ & 63,15 \\
\hline 13 & $-1,26$ & 0,69 & 62,80 & 0,68 & 0,06 & 63,21 \\
\hline 14 & $-1,86$ & 1,41 & 67,60 & 0,68 & $-0,70$ & 64,18 \\
\hline 15 & $-0,11$ & 1,34 & 63,29 & 0,29 & $-0,87$ & 63,32 \\
\hline 16 & 0,01 & 1,55 & 62,58 & 0,11 & $-0,66$ & 63,00 \\
\hline 17 & $-0,08$ & 1,52 & 62,96 & 0,17 & $-0,63$ & 63,28 \\
\hline 18 & $-0,09$ & 1,52 & 62,97 & 0,17 & $-0,63$ & 63,29 \\
\hline 19 & $-1,37$ & 0,64 & 62,82 & 0,68 & $-0,21$ & 63,04 \\
\hline 20 & $-0,47$ & 1,35 & 63,10 & 0,34 & $-0,51$ & 63,36 \\
\hline 21 & $-1,34$ & 0,65 & 62,88 & 0,68 & $-0,14$ & 63,16 \\
\hline 22 & $-0,21$ & 1,63 & 62,96 & 0,30 & $-0,88$ & 63,45 \\
\hline & & & & & &
\end{tabular}

Levando-se em conta que os parâmetros do centro de fase, quando negligenciados, produzem um maior impacto sobre a componente vertical das coordenadas de um ponto, apresenta-se, no gráfico da Figura 7, o módulo das diferenças entre as componentes verticais dos vetores PCOs propagados e dos vetores PCOs calibrados relativos a cada teste realizado. 


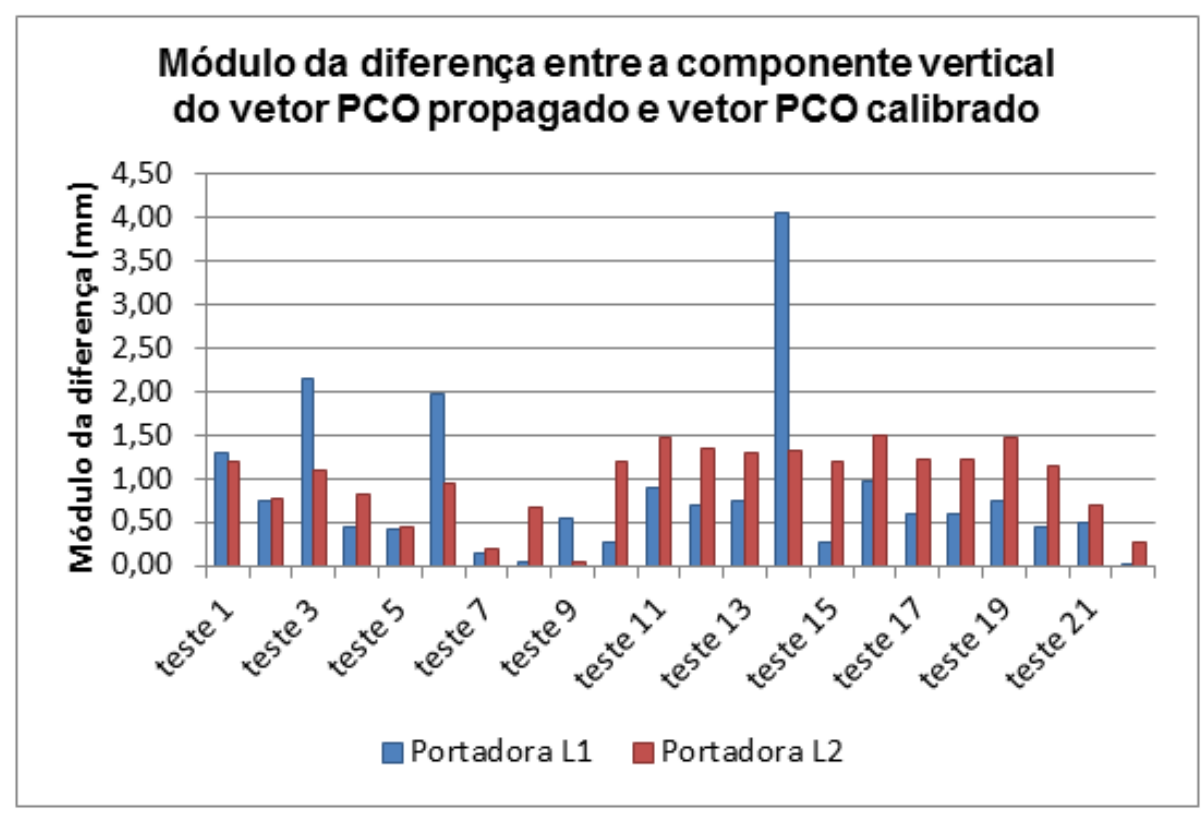

Figura 7: Diferenças entre as componentes verticais dos vetores PCOs propagados e calibrados.

Destacam-se os testes 3, 6 e 14, nos quais a diferença na componente vertical do vetor PCO para a onda portadora $\mathrm{L} 1$ atinge valores iguais a $2,15 \mathrm{~mm}, 1,97 \mathrm{~mm}$ e 4,05 $\mathrm{mm}$, respectivamente. Portanto, as maiores diferenças. No entanto, para a maioria dos testes, as discrepâncias não superam o valor de $1 \mathrm{~mm}$ para os testes com a onda portadora L1 e mantém-se abaixo de 1,5 $\mathrm{mm}$ para os testes com a onda portadora L2.

Associados aos parâmetros PCOs propagados, os parâmetros PCVs são determinados aplicandose a Equação 3. As maiores diferenças obtidas na comparação entre os parâmetros PCVs associados aos vetores PCOs propagados e os parâmetros PCVs associados aos vetores PCOs calibrados, em todos os testes, são apresentadas no gráfico da Figura 8.

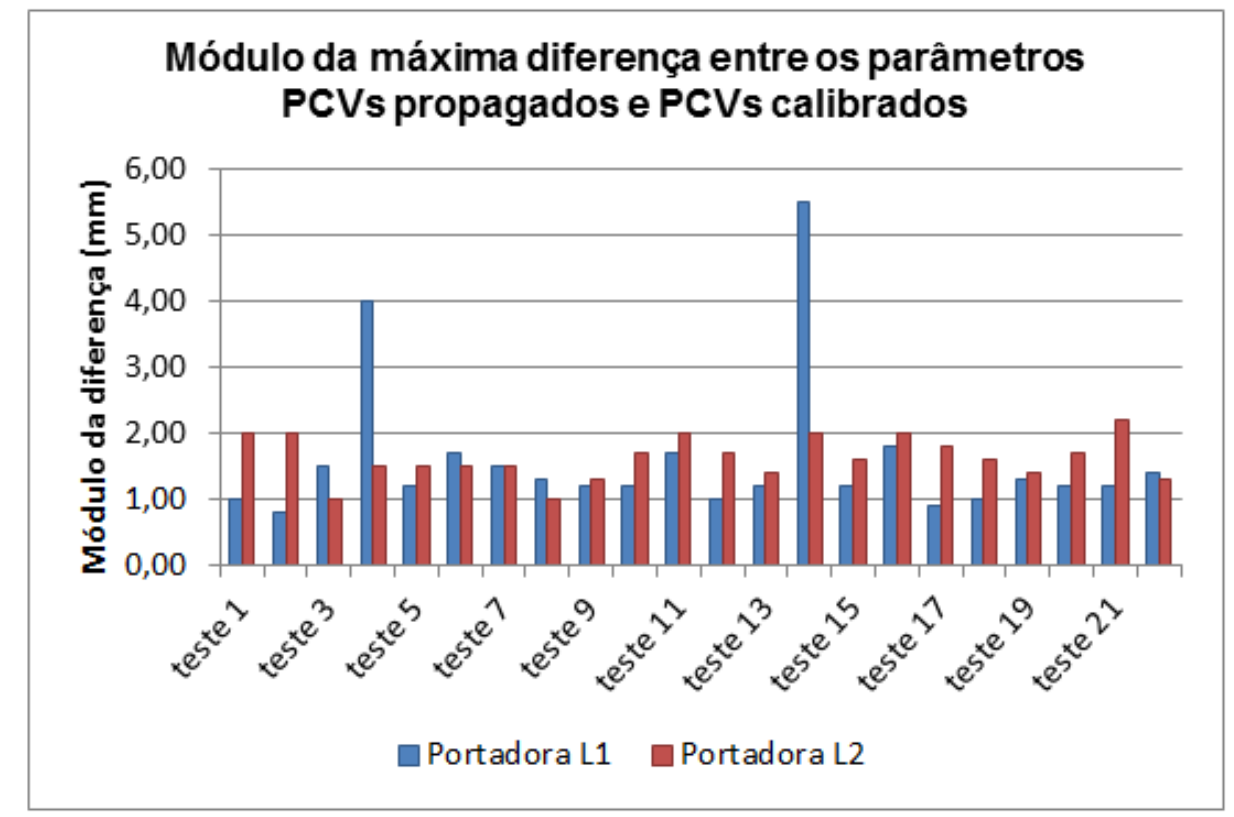

Figura 8: Diferenças máximas entre os parâmetros PCVs propagados e calibrados. 
Observa-se que as diferenças máximas obtidas nos testes 4 e 14 chegam a $4 \mathrm{~mm}$ e $5,5 \mathrm{~mm}$, respectivamente, considerando a onda portadora L1. Nos demais testes a maior diferença ocorre para o teste 21, atingindo o valor de $2,2 \mathrm{~mm}$, na portadora $\mathrm{L} 2$.

Diante das discrepâncias apresentadas pelos parâmetros PCOs e PCVs obtidos pela metodologia proposta em relação aos parâmetros calibrados, destacam-se os resultados dos testes 3, 4, 6 e 14 .

Analisando-se as componentes verticais dos vetores PCOs das campanhas primárias e secundárias dos respectivos testes, evidencia-se tanto a campanha 3 , utilizada no teste 14 , quanto a campanha 4, utilizada nos testes 3, 4 e 6, por apresentarem componentes verticais que se destacam das demais pelos seus valores referentes à onda portadora L1, 65,52 mm e 65,54 mm, respectivamente, Quadro 2 da subseção 3.2

Assim, foi verificado se tais parâmetros poderiam ter sido afetados por fatores relacionados ao clima. Após consulta aos registros das estações meteorológicas, constatou-se que houve períodos de precipitação durante o intervalo de tempo de realização da campanha de calibração 3 (14/05/2011) e campanha de calibração 4 (30/08/2011). Desta forma, a influência que se verifica nas campanhas primárias e/ou secundárias, deverá estar presente também nos parâmetros propagados.

Os valores médios e os desvios-padrão das componentes dos vetores PCOs propagados são calculados e comparados aos valores médios e desvios-padrão referentes aos vetores PCOs calibrados, determinados pelas 13 campanhas de calibração disponíveis. Os resultados são apresentados na Tabela 2.

Tabela 2: Medias e desvios-padrão dos parâmetros PCOs propagados e calibrados.

\begin{tabular}{|c|c|c|c|c|c|c|c|}
\hline & \multicolumn{3}{|c|}{ Portadora Ll } & \multicolumn{3}{|c|}{ Portadora L2 } \\
\hline & & $\mathbf{E}$ & $\mathbf{N}$ & $\mathbf{V}$ & $\mathbf{E}$ & $\mathbf{N}$ & $\mathbf{V}$ \\
\hline \multirow{2}{*}{$\begin{array}{c}\text { PCOs } \\
\text { calibrados }\end{array}$} & Média (mm) & $-0,66$ & 1,30 & 63,61 & 0,43 & $-0,48$ & 63,61 \\
\hline & $\begin{array}{c}\text { Desv. Padrão } \\
\text { (mm) }\end{array}$ & 0,51 & 0,34 & 0,99 & 0,17 & 0,25 & 0,53 \\
\hline \multirow{2}{*}{$\begin{array}{c}\text { PCOs } \\
\text { propagados }\end{array}$} & Média (mm) & $-0,57$ & 1,26 & 63,28 & 0,39 & $-0,54$ & 63,38 \\
\hline & $\begin{array}{c}\text { Desv. Padrão } \\
\text { (mm) }\end{array}$ & 0,61 & 0,41 & 1,20 & 0,22 & 0,29 & 0,46 \\
\hline
\end{tabular}

Os resultados foram submetidos ao teste de Kolmogorov-Smirnov (K-S), para a verificação de normalidade, e ao teste t de Student para uma média, com o objetivo de verificar se as diferenças apresentadas entre as médias dos parâmetros propagados e as médias dos parâmetros calibrados são significativas.

O teste K-S considera a máxima diferença absoluta entre a função de distribuição acumulada assumida teoricamente para os dados e a função de distribuição empírica, ou observada, para os dados. Como critério, compara-se esta diferença com um valor crítico, para um dado nível de significância (Conover, 1999). A Equação 8 expressa a diferença avaliada pelo teste K-S.

$$
D_{n}=\max \left|F(x)-F_{n}(x)\right|
$$

na qual $F(x)$ é a função acumulada teórica e $F_{n}(x)$ é a função acumulada observada. 
Como hipótese nula $\left(\mathrm{H}_{0}\right)$ assume-se que os valores pertencem a uma distribuição normal, com uma significância de $1 \%(\alpha=0,01)$. O valor crítico para o teste referente ao número de elementos da amostra é de 0,3367. A Tabela 3 apresenta os valores obtidos para o teste K-S.

Tabela 3: Teste Kolmogorov-Smirnov.

\begin{tabular}{c|c|c|c|c|c|c}
\cline { 2 - 7 } \multicolumn{2}{c|}{} & \multicolumn{3}{c|}{ Portadora L1 } & \multicolumn{3}{c}{ Portadora L2 } \\
\hline PCO & E & N & V & E & N & V \\
\hline Teste K-S & 0,3100 & 0,2571 & 0,3182 & 0,2264 & 0,1802 & 0,1899 \\
\hline
\end{tabular}

Para avaliar se a média dos parâmetros PCOs propagados é significativamente diferente em comparação à média dos parâmetros PCOs calibrados realizou-se o teste t para uma amostra que apresenta distribuição normal (Welcowitz et al., 2006). Como hipótese nula $\left(\mathrm{H}_{0}\right)$ estabelece-se que as médias para os parâmetros propagados não apresentam uma diferença significativa em relação à média dos parâmetros calibrados, a um nível de significância de 1\%. A hipótese alternativa $\left(\mathrm{H}_{1}\right)$ se caracteriza por considerar que a diferença entre as médias é significativa. A Tabela 4 apresenta os resultados para o teste t.

Tabela 4: Teste t de Student.

\begin{tabular}{c|c|c|c|c|c|c}
\cline { 2 - 7 } \multicolumn{3}{c|}{ Portadora L1 } & \multicolumn{3}{c}{ Portadora L2 } \\
\hline PCO & E & N & V & E & N & V \\
\hline Teste t & 0,6920 & $-0,4576$ & $-1,2898$ & $-0,8528$ & $-0,9704$ & $-2,3452$ \\
\hline
\end{tabular}

Como o teste conduzido é bilateral com $t_{\alpha / 2}=2,8314$ e os resultados indicam que $|t|<t_{a / 2}$, a hipótese nula não pode ser rejeitada, com uma significância de $1 \%$. Assim, considera-se que as médias dos parâmetros propagados não apresentam diferenças significativas quando comparadas às médias dos parâmetros calibrados.

\section{Conclusões}

Nas aplicações geodésicas que envolvem alta acurácia é importante conhecer com a máxima exatidão possível a posição do centro de fase da antena do receptor. O conhecimento completo do centro de fase da antena está relacionado ao estabelecimento dos parâmetros PCO e PCVs, mediante o processo de calibração da antena, exigindo, assim, o emprego de equipamentos específicos e locais adequados durante o intervalo de tempo em que os procedimentos são realizados.

Propõe-se que os parâmetros do centro de fase da antena sejam estimados por meio de integração numérica utilizando componentes do vetor PCO calibradas pelo programa WaSoft/Kalib. Os parâmetros propagados e calibrados foram comparados ao longo de 22 testes. 
Comparando-se as componentes verticais dos vetores PCOs propagados às respectivas componentes calibradas, as maiores diferenças, apresentadas pelos testes 3,6 e 14, relacionam-se com a utilização de parâmetros estabelecidos em campanhas de calibração sujeitas a períodos de precipitação. Para os demais testes as diferenças mantiveram-se abaixo de $1,5 \mathrm{~mm}$.

Com relação aos parâmetros PCVs, as maiores diferenças encontradas nas comparações com as PCVs originais são apresentadas pelos testes 4 e 14 e também estão relacionadas à campanhas em que ocorreram períodos de precipitação. As diferenças encontradas pelos demais testes apresentam um valor máximo de $2,2 \mathrm{~mm}$.

Os testes em que são utilizadas datas intermediárias na propagação estabelecida (testes 21 e 22), evidenciam o comportamento linear das componentes, produzindo discrepâncias submilimétricas na comparação entre as componentes verticais dos vetores PCOs propagados e calibrados.

Considerando apenas os testes em que são realizadas propagações baseadas em campanhas de calibração não afetadas por períodos de precipitação, as diferenças máximas chegam a 1,5 mm para os PCOs, 2,2 mm para as PCVs.

Através dos testes realizados e levando-se em consideração que as componentes do vetor PCO têm comportamento aproximadamente linear, possibilitando o desenvolvimento de um modelo matemático para a estimativa dessas componentes a partir de condições iniciais, propagando-se numericamente até uma data específica e determinando-se os valores das PCVs correspondentes, pode-se concluir que a metodologia proposta mostrou-se promissora.

O método proposto pela pesquisa deve ser levado em conta como uma alternativa viável, dependendo do nível de acurácia desejado, à estimativa de parâmetros calibrados do centro de fase da antena aplicando um modelo matemático baseado em integração numérica, utilizando parâmetros estabelecidos por duas campanhas de calibração não afetadas por precipitação.

Entretanto, a proposta apresentada não tem por objetivo substituir o procedimento de calibração da antena, uma vez que se trata de um modelo aproximado que pode ser levado em conta perante à necessidade de utilização de parâmetros calibrados da antena e a impossibilidade de realização de uma campanha de calibração para obtê-los.

\section{REFERÊNCIAS BIBLIOGRÁFICAS}

Balanis, C. A. Antenna Theory: Analysis And Design. 3rd edition. New York, John Wiley \& Sons, 2005.

Bartels, G. A. Series 08 Astrodynamics and Satellite Systems. Gps-Antenna Phase Center Measurements Performed in an Anechoic Chamber. The Netherlands, Delft University Press, 1997.

Born, M.; Wolf, E. Principles of Optics: Electromagnetic Theory of Propagation Interference and Diffraction of Light. 6th edition. Great Britain, Cambridge University Press, 1980.

Conover, W. J. Practical Nonparametric Statistics. 3rd edition. United States of America, John Wiley \& Sons, 1999.

De Jong, G. "The Phase Center of a Monopole Antenna." Radio Science 17, no. 2 (1982): 349 55. Accessed May 29, 2014. doi 10.1029/RS017i002p00349. 
El-Hattab, A. I. "Influence of GPS Antenna Phase Center Variation on Precise Positioning." NRIAG Journal of Astronomy and Geophysics 2 (2013): 272 - 77. Accessed May 28, 2014. doi 10.1016/j.nrjag.2013.11.002.

Fang, P.; Bevis, M.; Bock, Y.; Gutman, S.; Wolfe, D. "GPS Meteorology: Reducing Systematic Errors in Geodetic Estimates for Zenith Delay." Geophysics Research Letters 25, no. 19 (1998): $3583-86$.

Freiberger Junior, J. "Investigação da Calibração Relativa de Antenas GNSS." Tese de Doutorado, Universidade Federal do Paraná, 2007.

Görres, B.; Campbell, J.; Becker, M.; Siemes, M. "Absolute Calibration of GPS Antennas: Laboratory Results and Comparison with Field and Robot Techniques." GPS Solutions 10 (2006): 136 - 45. Accessed March 19, 2012. doi 10.1007/s10291-005-0015-3.

Hecht, E. Optics. 2nd edition. Reading, Addison-Wesley Publishing Company, 1990.

Huinca, S. C. M. "Calibração Relativa de Antenas GNSS na BCAL/UFPR.” Dissertação de de Mestrado, Universidade Federal do Paraná, 2009.

Huinca, S. C. M.; Krueger, C. P.; Mayer, M.; Knöpfler, A.; Heck, B. "First Results of Relative Field Calibration of a GPS Antenna at BCAL/UFPR." In Proceedings of the International Association of Geodesy Symposia. Geodesy for Planet Earth, Buenos Aires, 2012, 739-744.

Huinca, S. C. M. "Análise de Influências Ambientais na Calibração de Antenas de Receptores GPS.” Tese de Doutorado, Universidade Federal do Paraná, 2014.

Institute of Electrical and Electronics Engineers (IEEE). IEEE Standard Definitions of Terms for Antennas. New York, IEEE, 1983.

Kraus, J. D. Antenas. Rio de Janeiro, Guanabara Dois, 1983.

Krueger, C. P.; Freiberger Junior, J.; Heck, B.; Mayer, M.; Knöpfler, A.; Schäfer, B. "Establishing a GNSS Receiver Antenna Calibration Field in The Framework of PROBAL." In: Proceedings of the International Association of Geodesy Symposia. Observing Our Changing Earth, Peruggia, 2007, 701-708.

Krueger, C. P.; Huinca, S. C. M.; Leandro, D.; Viski, A. R. "Inovações Tecnológicas do Laboratório de Geodésia Espacial e Hidrografia (LAGEH)." Artigo apresentado no IV Simpósio Brasileiro de Ciências Geodésicas e Tecnologias da Geoinformação, Brasil, Recife, Maio 0609, 2012.

Mader, G. L. "GPS Antenna Calibration at the National Geodetic Survey. GPS Solutions 3 no. 1, (1999): 50 - 58. Accessed March 19, 2012. doi 10.1007/PL00012780.

Markov, G. Antennas. Moscow, Progress Publishers, 1965.

Menge, F.; Seeber, G.; Völksen, C.; Wübbena, G.; Schmitz, M. "Results of Absolute Field Calibrations of GPS Antenna PCV." Paper presented at the International Technical Meeting of the Satellite Division, ION GPS-98, Nashville, Tennessee, September 15-18, 1998.

Menge, F. "Zur Kalibrierung der Phasenzentrumsvariationen von GPS-Antennen für die Hochpräzise Posistionsbestimmung.” Phd diss. Universität Hannover, 2003. 
Monico, J. F. G. Posicionamento pelo GNSS: Descrição, Fundamentos e Aplicações. São Paulo, Editora UNESP, 2008.

Morescki Junior, L. F. M.; Ferreira, L. D. D. "Determinação dos Coeficientes dos Harmônicos Esféricos Para o Cálculo dos Parâmetros PCV e PCO Utilizando Dados de Programas Comerciais de Calibração." Boletim de Ciências Geodésicas 20, no. 2 (2014) 444-66. doi 10.1590/S1982-21702013000400026.

National Aeronautics and Space Administration. Classical Fifth-,Sixth -, Seventh - and EightOrder Runge-Kutta Formulas with Step Size Control. By Erwing Fehlberg. NASA TR R-287. Washington, D.C.: United States of America, 1968.

Rothacher, M.; Schaer, S.; Mervat, L.; Beutler, G. "Determination of Antenna Phase Center Variations Using GPS Data." Paper presented at the IGS Workshop, Potsdam, Germany, May 15-17, 1995.

Seeber, G. Satellite Geodesy: Foundations, Methods and Aplications. Berlin. Walter de Gruyter, 1993.

Welcowitz, J.; Cohen, B. H.; Ewen, R. B. 6th edition. Introductory Statistics for the Behavioral Sciences. New Jersey. John Wiley \& Sons, 2006.

Zeimetz, P.; Kuhlmann, H. "On the Accuracy of Absolute GNSS Antenna Calibration and the Conception of a New Anechoic Chamber." Paper presented at the GNSS Antenna Calibration and Accuracy Assessment Working Week, Stockholm, Sweden, June 14-19, 2008.

Recebido em Abril de 2015.

Aceito em Junho de 2015. 